\title{
Overexpression of CD44v8-10 in Colon Polyps-A Possible Key to Early Diagnosis
}

Milan Dastych ${ }^{1}$, Frantisek Hubatka ${ }^{2,3}$, Pavlina Turanek-Knotigova ${ }^{2,3}$, Josef Masek $^{2}$,
Radek Kroupa ${ }^{1}$, Milan Raška $^{4}$, Jaroslav Turanek ${ }^{2,3,5,6 *}$ and Lubomir Prochazka ${ }^{2 *}$

${ }^{1}$ Department of Gastroenterology and Internal Medicine, University Hospital Brno and Faculty of Medicine Masaryk University Brno, Brno, Czech Republic, ${ }^{2}$ Department of Pharmacology and Toxicology, Veterinary Research Institute, Brno, Czech Republic, ${ }^{3}$ C2P NEXARS, Campus Science Park, Brno, Czech Republic, ${ }^{4}$ Department of Immunology, Faculty of Medicine and Dentistry, Palacky University Olomouc, Olomouc, Czech Republic, ${ }^{5}$ Faculty of Medicine in Hradec Kralove, Institute of Hygiene and Preventive Medicine, Charles University, Hradec Kralove, Czech Republic, ${ }^{6}$ Institute of Physics of the Czech Academy of Sciences, Prague 8, Czech Republic

Background and aims: The majority of colorectal cancers arise from detectable adenomatous or serrated lesions. Here we demonstrate how deregulated alternative splicing of CD44 gene in diseased colon mucosa results in downregulation of standard isoform of CD44 gene (CD44s) and upregulation of variant isoform CD44v8-10. Our aim is to show that upregulation of CD44v8-10 isoform is a possible marker of precancerous lesion in human colon.

Methods: We analysed pairs of fresh biopsy specimen of large intestine in a cohort of 50 patients. We studied and compared alternative splicing profile of CD44 gene in colon polyps and adjoined healthy colon mucosa. We performed end-point and qRT PCR, western blotting, IHC staining and flow cytometry analyses.

OPEN ACCESS

Edited by:

László Kopper,

Semmelweis University, Hungary

*Correspondence: Jaroslav Turanek turanek@seznam.cz Lubomir Prochazka

lubomir.pr@gmail.com

Received: 05 October 2020 Accepted: 26 February 2021

Published: 30 March 2021

Citation:

Dastych M, Hubatka F, Turanek-Knotigova P, Masek J, Kroupa R, Raška M, Turanek J and Prochazka $L$ (2021) Overexpression of CD44v8-10 in Colon Polyps $-A$ Possible Key to Early Diagnosis. Pathol. Oncol. Res. 27:614281. doi: 10.3389/pore.2021.614281
Results: We detected more than five-fold overexpression of CD44v8-10 isoform and almost twenty-fold downregulation of standard isoform CD44s in colon polyps compared to adjoined healthy tissue with $p=0.018$ and $p<0.001$ in a cohort of 50 patients. Our results also show that aberrant splicing of CD44 occurs in both biologically distinct subtypes of colorectal adenoma possibly in ESRP-1 specific manner.

Conclusion: $92 \%$ of the colon polyp positive patients overexpressed CD44v8-10 isoform in their colon polyps while only $36 \%$ of them had positive fecal occult blood test which is currently a standard non-invasive screening technique.

Impact: We believe that our results are important for further steps leading to application of CD44v8-10 isoform as a biomarker of colorectal precancerosis in non-invasive detection. Early detection of colon precancerosis means successful prevention of colorectal carcinoma.

Keywords: CD44 isoforms, colorectal precancerosis, colon polyps, cancer markers, RNA splicing

Abbreviations: ASP, alternative splicing profile; SR-protein, serine arginine rich RNA binding protein; ESRP, epithelial cell type specific splicing regulators; CD44s, standard isoform of CD44; CD44v, variable isoform of CD44. 


\section{INTRODUCTION}

Colorectal cancer (CRC) is the second leading cause of cancer related death in Europe [1] and fourth and third in males and females worldwide [2]. The basic mechanism of colorectal tumorigenesis is quite straightforward stepwise process from precancerous to malignant stage $[3,4]$ although molecular processes during tissue remodeling and tumor microenvironment interaction remain under intensive research. CRC is preventable partly due to early detection of preneoplastic or neoplastic lesions in the large intestine unlike most of other cancers. Regular screenings mainly colonoscopies and fecal occult blood testing significantly increased the ratio of early detected CRC patients and proved to be effective [5]. Possible early detection of precancerous lesions through CD44 receptor became the focus of our study. CD44 receptor is expressed widely throughout the human body in both standard and variable isoforms [6]. CD44 receptor has several physiological functions like cell adhesion through binding of hyaluronic acid, the main component of extracellular matrix $[7,8]$ and participation in signal transduction and apoptosis control [9].

Since 1990's it was discovered that CD44 transcript is a subject of alternative splicing that generates various isoforms of CD44 (Figure 1A). Alternative splicing of CD44 is tissue specific and often deregulated in cancer cells. Initial studies suggested that some isoforms of CD44 receptor, e.g., CD44v6 play important role in the process of metastasizing. Moreover, total amount of CD44 receptor detected with pan-CD44 antibodies was introduced as a marker of presence of tumour initiating cells regardless its splicing variability described later on [10]. Despite extensive research on CD44 and its isoforms (CD44v) its role as a marker of malignancy or patient's prognosis remains unclear. However, it is clear that expression profile of CD44 changes as tumour develops. As mentioned above alternative splicing generates various isoforms of CD44 that appear and disappear at various stages of cancer development. This process of deregulated alternative splicing of CD44 starts already in adenomatous and hyperplastic epithelium.

$\mathrm{CD} 44 \mathrm{v}$ isoforms play various roles in cancer that may have high or low impact on the key features of cancer cells like metastasizing or tumor initiating potential. It is concluded that tumors expressing certain isoforms of CD44v are more aggressive compared to tumors expressing CD44s-only [6]. CD44 isoform switching in cancer cells as well as normal cells happens in response to some stimuli like oxidative stress [11], activation of signal transduction pathways especially MAP kinases [12, 13], epigenetics [14] or upon activation of resting lymphocytes [15]. It is apparent that CD44 isoform switching mediated by alternative splicing of CD44 has an impact on epithelial-mesenchymal transition [16] and similarly on metastatic behavior of cancer cells [17-20].

CD44 has attracted unusual attention from scientist since it was shown that particular CD44v isoform appear in cancer cells during tumor progression. CD44v6 was one of the first isoforms that aspired to become a biomarker due to the studies showing its prometastatic effect in rats [20]. Moreover, anti-metastatic effect of antibodies raised against CD44v6 isoform supported CD44v6 as a putative biomarker of malignancy [18]. Later studies showed that the predictive role of CD44 and CD44v differs among tissues and that each tissue needs its own detailed study. Cancer stem cell model and a discovery of CD44 as a marker of tumor initiating breast cancer cells [10] further extended attractiveness of CD44. CD44 and its selected variants were further reported as markers

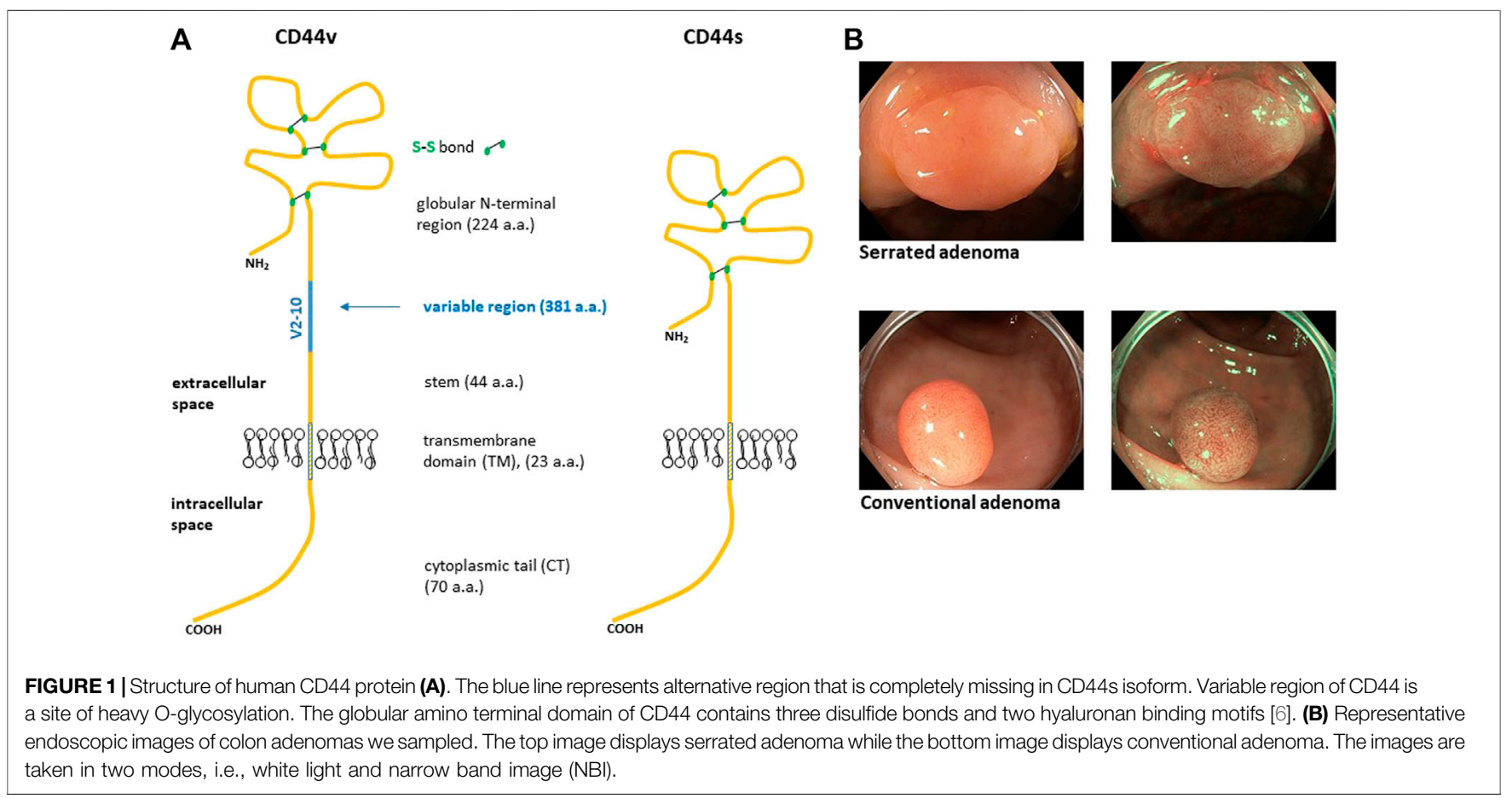


TABLE 1 | Clinical significance of various CD44 isoforms in certain cancer types. A reduced list of CD44 prognostic markers in various cancer types. met., metastasis; neg., negative.

\begin{tabular}{|c|c|c|}
\hline Cancer type & Isoform & Clinical significance \\
\hline Lung cancer & CD44v6, CD44s & Metastasis, survival $[22,23]$ \\
\hline Breast cancer & Pan-CD44 (all isoforms) & Differentiation, neg. prognosis $[10,23]$ \\
\hline Prostate cancer & CD44v6, $\downarrow$ CD44s & Met., prognosis $[23,24]$ \\
\hline Colorectal cancer & $\downarrow C D 44 s$, CD44v6, CD44v9 & Met., prognosis [23, 25, 26] \\
\hline Bladder cancer & CD44v8-10 & Neg. prognosis [27] \\
\hline
\end{tabular}

of poor prognosis in gastric cancer patients. CD44v8-10 was later identified as a gastric cancer stem cell marker [21]. Generally, altered expression of CD44s and CD44v in various cancer types might have a certain prognostic value (see examples in Table 1).

Fewer than $10 \%$ of colon adenomas progress to cancer [28]. Risk of progression to carcinoma is higher if villous architecture or high grade dysplasia is present. Colorectal carcinoma is preceded by pre-invasive stage of neoplasia that lasts for years and is typically asymptomatic [29]. Early detection of CRC could be possible through searching for biomarkers or markers of cancer cells [30].

In our study, we explored alternative splicing of CD44 directly in human precancerosis. We studied expression of variant isoforms of CD44 in precancerous tissue of the large intestine and compared the level of expression of CD44 in adjacent healthy mucosa in a cohort of 50 colorectal adenoma patients. We detected constant repetition of splicing and expression profile of CD44 receptor in the patients participating in our study. Our study puts CD44 receptor into the context of diagnosis of precancerosis rather than prognosis of CRC.

\section{MATERIALS AND METHODS}

\section{Cell Culture}

Primary Human Epidermal Keratinocytes cells (Cell Applications, Inc.) were cultured in KGM-Gold ${ }^{\mathrm{TM}}$ medium with SingleQuots ${ }^{\mathrm{TM}}$ growth supplements (Lonza). HEL 12469-Human embryonic lung fibroblast (ECACC) were cultured in DMEM with $2 \mathrm{mM}$ Glutamine (Healthcare Life Science HyClone) 1\% Non Essential Amino Acids (GE Healthcare Life Science HyClone) and 10\% Fetal Bovine Serum (GE Healthcare Life Science HyClone). The p53deficient H1299 non-small cell lung cancer cell line was grown in RPMI-1640 medium supplemented with antibiotics and 10\% FBS. Human breast carcinoma T47D cell line was grown in RPMI-1640 medium supplemented with antibiotics and 10\% FBS. The cells were grown in a petri dish and detached at $80-90 \%$ confluency using accutase prior to incubation with HA-FITC.

\section{Endoscopic Screening and Sampling}

Colonoscopy and mucosal sampling were performed in the Clinic of Internal Medicine-Hepatogastroenterology, University Hospital Brno using medical video endoscopes Olympus CF HQ190L, EVIS EXERA III. Endoscopic polypectomy and mucosectomy were proceed by standard technique using endoscopic needle, snare and electrosurgical unit (Interjet Needle Con $25 \mathrm{G} / 4 \mathrm{~mm} / 240 \mathrm{~cm}$ Boston, Dis Micro oval Snare $13 \mathrm{~mm}$ Boston, SD-990-15 HF polyp snare Olympus, VIO 300D ERBE). The tissue samples were collected using standard biopsy forceps (Radial Jaw 4 SC w/NDL, Boston). Samples for the purposes of our study were snap-frozen immediately after biopsy in isopropanol pre cooled on dry ice. This project is approved by the ethical committee of the University Hospital Brno. Each patient signed informed consent and participated voluntarily in our project. All experiments were performed in accordance with relevant guidelines and regulations.

\section{Real-Time and End-point PCR}

Defrosted biopsy specimen was placed into $7 \mathrm{ml}$ all glass Tissue grinder (Kimble Kontes). The homogenizer was then placed into liquid nitrogen for several seconds until the tissue froze and got fragile. The frozen tissue was then crushed using pestle B. The crushed tissue was then dissolved in lysis/binding buffer (Roche) and transferred into an eppendorf tube using PTFE tubing and $1 \mathrm{ml}$ syringe. Total RNA from biopsy specimen was then isolated using High Pure RNA Tissue Kit (Roche, Cat. No. 12033674 001) according to the manufacturers protocol. Quality and concentration of isolated RNA was checked using NanoDrop2000 (ThermoFisher). Isolated RNA was then transcribed to cDNA using oligo dT primers (Invitrogen, Cat. No. 18418-020) and the M-MLV reverse transcriptase (Invitrogen Cat. No. 28025-013). End-point PCR was performed using Master Mix PCR, $2 \mathrm{mM}$ (Generi Biotech, Czech Republic). $500 \mathrm{ng}$ of cDNA and $0.25 \mu \mathrm{M}$ concetration of the primers was used. Actin was used as a loading control. Endpoint PCR primers were as follows. CD44v3-c19: Forward 5' AGGCTGGGAGCCAAATGAAG-3', Reverse 5' -TGGGGTGGA ATGTGTCTTGG-3'; CD44v4-c19: Forward 5' - ACGGGCTTT TGACCACACAA- ${ }^{\prime}$, Reverse $5^{\prime}$-TGGGGTGGAATGTGTCTT GG-3'; CD44v5-c19: Forward 5'-CACACCCTCCCCTCATTC AC- $3^{\prime}$, Reverese $5^{\prime}$-GCTCCACCTTCTTGACTCCC-3'; CD44v6-c19: Forward $5^{\prime}$-GTGGTTTGGCAACAGATGGC-3', Reverse $5^{\prime}$-GCTCCACCTTCTTGACTCCC-3'; CD44v7-c19: Forward $5^{\prime}$-GGACGAGGTCATCAAGCAGG-3', reverse $5^{\prime}$ GGGGTGGAATGTGTCTTGGT-3'; CD44v8-c19(8v8): Forward 5'-AACGCTTCAGCCTACTGCAA-3', Reverse 5'TGGGGTGGAATGTGTCTTGG-3'; CD44v9-c19: Forward $5^{\prime}$ ACATCACATGAAGGCTTGGA-3', Reverse $5^{\prime}$-GCTCCACCT TCTTGACTCCC-3'; CD44v10-c19: Forward 5'-ACACGAAGG AAAGCAGGACC-3' ${ }^{\prime}$, Reverse $5^{\prime}$ - GCTCCACCTTCTTGACTC 
CC-3'; CD44s(CD44s1): Forward 5'-AGTCACAGACCTGCC CAATG-3', Reverse $5^{\prime}$-GCTCCACCTTCTTGACTCCC-3'; Actin: Forward $5^{\prime}$-AATCTGGCACCACACCTTCT-3', Reverse 5'-AGCACAGCCTGGATAGCAAC-3'; Quantitative real-time PCR (qPCR) was performed using the LightCycler 480 instrument (Roche) and The LightCycler ${ }^{\circledast} 480$ SYBR Green I Master (Roche). $200 \mathrm{ng}$ of cDNA and $0.1 \mu \mathrm{M}$ concentration the primers was used for PCR reaction. Relative quantification of target genes expression was performed using the formula described elsewhere [31]. PCR primers were as follows. Actin: Forward 5'-AATCTGGCACCACACCTTCT-3', Reverse 5'AGCACAGCCTGGATAGCAAC-3'; 5 + v8 des3: Forward 5'CCAGTGAAAGGAGCAGCACT- ${ }^{\prime}$, Reverse $5^{\prime}$-AGTCCATAT TGGTAGCAGGGA-3'; 5 + 15 CD44s J4: Forward 5'-AAGGAG CAGCACTTCAGGAG- $3^{\prime}$, Reverse $5^{\prime}$-TGTGTCTTGGTCTCT GGTAG-3'; ESRP-1: Forward 5'-CAGAGGCACAAACATCAC AT-3', Reverse 5' -AGAAACTGGGCTACCTCATTGG-3'; ESRP-2: Forward 5'-GACGTGCTTGGCTTCCT-3', Reverse 5'-AGCTCCTCACAAGCAAAGAG-3'; Sam68: Forward 5'ACCGTAAACCAGTCGGCATC-3' ${ }^{\prime}$, Reverse $5^{\prime}$-CGTCGTCTC CCCATTCTCAT-3'; TRA2B: Forward $5^{\prime}$-CTACGGCGAGCG GGAATC- ${ }^{\prime}$, Reverse $5^{\prime}$-GACTTTGATCTGGAACGCCT- $3^{\prime}$; SFRS6: Forward 5'-CTGCGTCGTATCAAGCGTTTT-3', Reverse 5'-TGTATCAGCCAGTTGACTCCG-3';

\section{Western Blot Analysis}

Whole cell lysis (WCL) buffer was used to dissolve crushed tissue. WCL formulation was $10 \mathrm{mM}$ Tris at $\mathrm{pH} 7.4,1 \mathrm{mM} \mathrm{NaF}, 1 \mathrm{mM}$ $\mathrm{Na}_{3} \mathrm{VO}_{4}, 1 \mathrm{mM}$ PMSF, $0.3 \%$ SDS, $1 \%$ Triton X-100 and the protease inhibitor cocktail [32]. The crushed tissue was dissolved in WCL buffer and transferred into an eppendorf tube and sonicated on ice. The lysate was then spun down at $16,000 \times g$ for $3 \mathrm{~min}$, and the resulting supernatant was used for sample preparation by mixing with $2 \mathrm{x}$ or $4 \mathrm{x}$ concentrated Laemmli buffer and boiled for $4 \mathrm{~min}$. An aliquot of the lysate was used for Protein level assay using BCA method (Pierce). Proteins were resolved by SDS/PAGE and transferred to PVDF membranes (GE Healthcare). After probing with a specific primary antibody and horse radish peroxidise (HRP)conjugated secondary antibody, the protein bands were detected with the ECL kit using X-ray medical film (Kodak). The antibodies used were anti-actin IgG (AC-15, Sigma), antipan CD44 IgG (2C5 BBA10, R\&D Systems), anti-CD44v10 IgG (AB2082, Millipore), anti-mouse IgG-HRP (A4416, Sigma), antirabbit IgG-HRP (NA934VS, GE Healthcare).

\section{Immunohistochemistry Staining-Fluorescent Detection}

The snap-frozen tissues were sectioned to $8-10 \mu \mathrm{m}$ slices using cryocut Leica 900 at $-15^{\circ} \mathrm{C}$ and mount on gelatin coated histological slides. The tissue sections were then fixed using pre cooled acetone for $5 \mathrm{~min}$ and stored in the freezer for IHC analysis. The sections were defrosted in humidity chamber and washed $2 \times 5 \mathrm{~min}$ in TBS $0.025 \%$ Triton- 100 . The sections were then blocked in $10 \%$ goat serum with $1 \%$ BSA in TBS for $1-2 \mathrm{~h}$ at room temperature. The slides were drained for a few seconds and wiped around the sections with tissue paper. Primary antibody was diluted in TBS with $1 \%$ BSA at $0.5 \mu \mathrm{l} / 100 \mu \mathrm{l}$ and applied for $1 \mathrm{~h}$. The slides were then washed $3 \mathrm{x}$ times in TBS $0.025 \%$ Triton100. Fluorophore-conjugated secondary antibody diluted in TBS with $1 \% \mathrm{BSA}$ at $1 \mu \mathrm{l} / 100 \mu \mathrm{l}$ for $1 \mathrm{~h}$ was applied on the slide. $10 \mathrm{~min}$ before washing $100 \mu \mathrm{l}$ of $0.3 \mu \mathrm{l} \mathrm{DAPI} / 100 \mathrm{ul}$ TBS with $1 \% \mathrm{BSA}$ was mixed in. The slides were then washed $3 \times 5 \mathrm{~min}$ in TBS, mounting medium was added and coverslip placed. The slides were observed using epifluorescent microscope Nikon. We used anti-CD44 IgG (2C5, R\&D Systems), goat anti-mouse FITC (SCBT).

\section{Flow Cytometry}

The tissue samples used for HA binding test were stored in cold PBS prior to accutase extraction. Extraction of primary cells from adenomatous and healthy tissue was performed using the enzyme accutase. The tissue digest suspension containing colonocytes as well as stromal cells was left for $24 \mathrm{~h}$ in D-MEM serum free culture medium supplemented with Insulin $5 \mathrm{U} / \mathrm{ml}$. The cells were then scraped off the petri dish using rubber policeman and washed twice with PBS. The cell suspension was then incubated for $15 \mathrm{~min}$ with fluorescently labeled Hyaluronic acid (HA-FITC, Sigma Aldrich) in serum free culture medium and finally washed once with PBS. The fluorescence intensity was then measured using flow cytometry (FACS Calibur, BD Bioscience).

\section{Statistics}

Unless otherwise stated, data were analysed using the GraphPad PRISM 5.00 software, and represent mean \pm SD of three independent experiments. Images are representative of at least three independent experiments. Relative quantification of target genes expression was performed using the formula described elsewhere [31]. Column statistics $t$ test against hypothetical value of 1.0 was then used for overall statistical analysis of the whole group of patients.

\section{RESULTS}

Our previous research on CD44 receptor lead us to analyse alternative splicing profile (ASP) of $\mathrm{CD} 44$ gene in colon polyps. We originally observed a difference in ASP of CD44 between adenomatous and healthy colon mucosa in several tissue samples obtained from the University Hospital Brno. Consistent splicing differences between colon polyps and healthy adjacent mucosa throughout the pilot samples and the lack of exploration of ASP in colon precancerosis made us gradually extend the number of patients up to a cohort of 50 patients. The patients involved in our study were randomly selected during regular colonoscopy screenings. Fifty patients having conventional or serrated adenoma contributed with a biopsy specimen of their lesion and adjacent healthy mucosa (Figure 1B). Serrated adenoma $n=12$, tubular adenoma $n=28$, tubulovillous adenoma $n=10$.

We used end-point PCR to describe the overall alternative splicing profile (ASP) of CD44 in colon polyps and in adjacent healthy mucosa. PCR primers covered the whole variable part of 


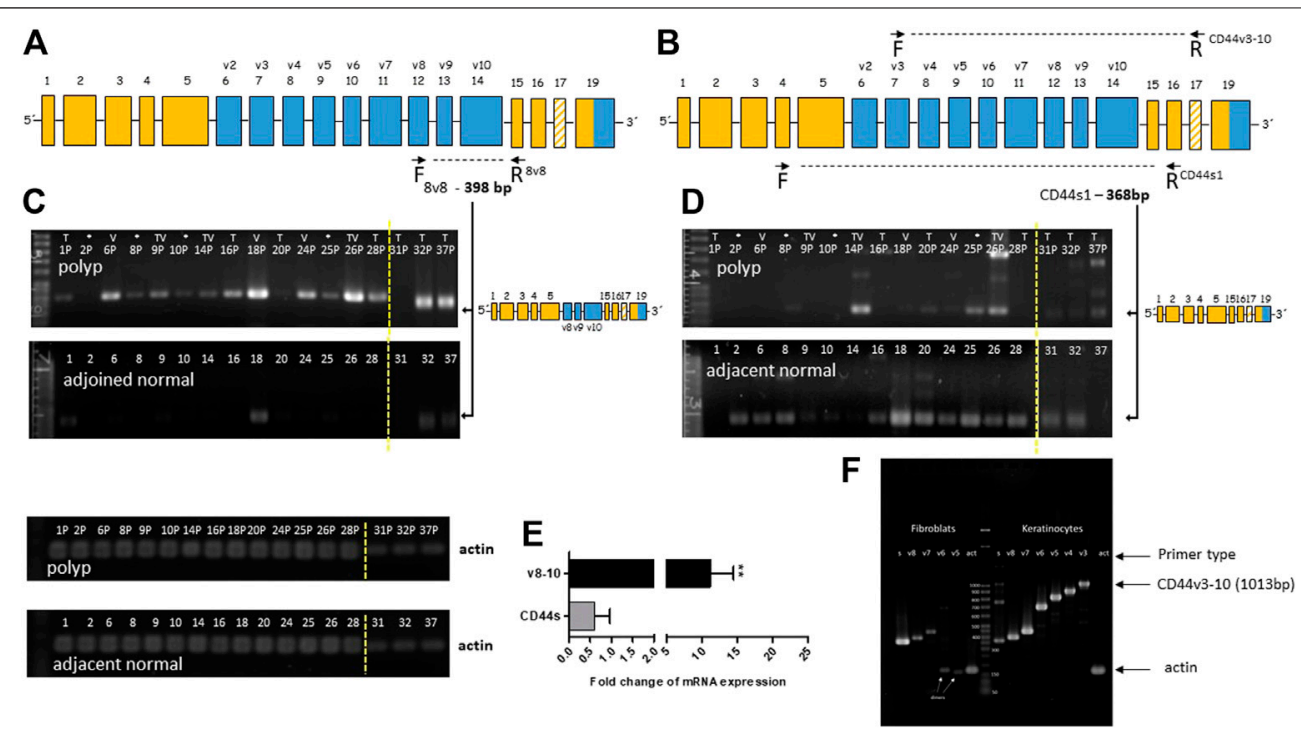

FIGURE 2 | Overexpression of CD44v8-10 in colon polyps (A,B) Position of the selected primers in CD44 mRNA exon structure. Constant exons (always transcribed) are marked orange. Variable exons are marked blue. (A) 8v8 primers were used for detection of inclusion of variable exons v8-10, i.e., CD44v8-10. Product size of 398 bp confirms transcription of variable exons v8-10. (B) CD44s1 primers span the whole variable region of the CD44 gene. Product size of $368 \mathrm{bp}$ relates to the standard isoform of CD44 (CD44s). Product size > 368 bp indicates inclusion of variable exons. (C,D) Agarose gel resolution of end-point PCR products of 8 v8 primers (C) and CD44s1 primers (D) in polyp and healthy tissue samples. Actin was used as a loading control. PCR products for actin are displayed below. The arrows indicate product representing CD44v8-10 (C) and CD44s (D). Relative quantification using densitometric analysis of agarose gels is represented by the bar graph (E). An agarose gel after resolution of the PCR products of CD44v3 primers that start at exon $\mathrm{v} 3$ and finish at the constant region (F). Keratinocytes were used as a positive control for exon inclusion while fibroblast express mostly CD44s. Increasing size of PCR products means higher number of variable exons included in the final mRNA transcript. Yellow dashed line delineates grouping of two gels into one picture. Samples from patients with sessile serrated adenoma (SSA) are marked with asterisk ( $\left.{ }^{\star}\right)$; samples of villous adenomas are marked (V); samples of tubular adenomas are marked (T); samples of tubulovillous adenomas are marked (TV).

the gene (marked blue Figure 2A,B). Verification of our endpoint PCR set up was done using human primary keratinocytes that express all major isoforms of CD44v. We detected and displayed all major isoforms of CD44, CD44v (Figure 2F). We confirmed that our PCR method works well, identifies all major $\mathrm{CD} 44 \mathrm{v}$ isoforms and is therefore a good indicator of aberrant splicing. Alternative splicing profile of CD44 in colon polyps differed substantially from alternative splicing profile of CD44 in adjacent healthy mucosa (Figures 2C,D). DNA electrophoresis showed that the main product of CD44s1 primers was size of $368 \mathrm{bp}$ and corresponded to the standard isoform of CD44, i.e., CD44s was found in all samples of healthy tissue (Figure 2D). The same CD44s1 primers produced just a weak band of this size in less than $50 \%$ of polyp samples. Interestingly, products of higher size than $368 \mathrm{bp}$ that respond to inclusion of variable exons were observed in polyp samples. We thus designed specific primers that coded products starting in particular variable region and ending in the constant region of CD44 gene, i.e., starting from exons v2-v10 and ending in C6, e.g., v8-C6 as shown in Figure 2A. We tested primary fibroblasts in order to have a negative control as well as to estimate any false positive contribution of fibroblast contamination to the level of variable isoforms in our lysates (Figure 2F). Agarose gels resolving PCR products of healthy tissue differed significantly from those resolving PCR products of polyp tissue. The gels representing polyp samples showed mainly PCR products of primers specific for v8, v9 and v10 exons while gels representing healthy tissue were blank. The molecular weight of PCR products confirmed theoretical product size of CD44v8-10 isoform. The end-point PCR data clearly indicated upregulation of CD44v8-10 isoform in polyp samples (Figures 2C,E).

We subsequently performed real-time PCR to evaluate the overexpression of the polyp specific CD44v8-10 isoform. Previous end-point PCR experiments confirmed that v8 isoform is included only in v8-10 transcript. We designed primers spanning exon junction of constant exon $\mathrm{C} 5$ and variable exon v8 while antisense primer was complementary to the C5 constant exon (Figure 3A). The real time PCR analysis of 50 patients confirmed more than five-fold overexpression of CD44v8-10 isoform with $p=0.018$ in colon polyps compared to adjacent healthy tissue (Figure 3D). 92\% of patients had upregulated expression of CD44v8-10 in their polyps. Realtime PCR using primers specific for CD44s, i.e., primers spanning exon junction of $\mathrm{C} 5+\mathrm{C} 15$ Figure $3 \mathbf{B}$ revealed that $\mathrm{CD} 44 \mathrm{~s}$ is downregulated by twenty-fold in colon polyps compared to healthy tissue with $p<0.001$ (Figure 3D). In other words, $100 \%$ of patients involved in our study had downregulated expression of CD44s in their precancerous lesions compared to their healthy adjacent counterparts. The size of real-time PCR products was also checked using agarose gel electrophoresis (Figure 3C).

Mouse anti-total CD44 IgG proved that CD44s is strongly downregulated also at the protein level in colon polyps (Figure 4). 


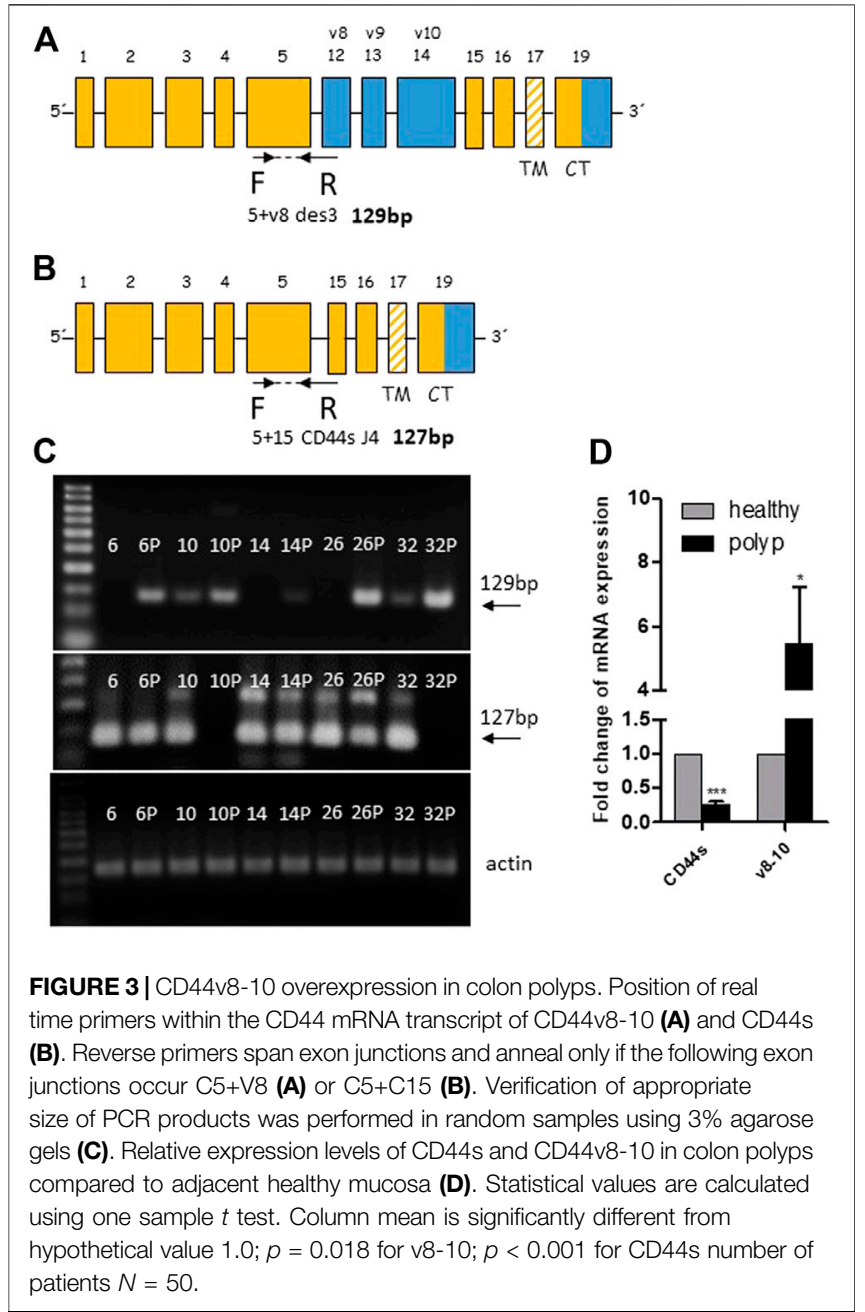

Not surprisingly, immunoblotting with rabbit anti-CD44v10 IgG that also recognizes CD44v8-10 [33] showed that polyp biopsies contain significantly larger amounts of CD44v8-10 isoform (Figure 4). This indicates that translation correlates with RNA expression.

To avoid any possible false positive results we decided for immunohistochemical staining of polyp tissue sections. It is known that fibroblasts express low levels of CD44v8-10 (Figure 2F). We identified the site of expression of CD44s and $\mathrm{CD} 44 \mathrm{v}$ in tissue sections of colon polyps. The tissue sections were immunolabeled using combination of primary and fluorescently labeled secondary antibodies. The mouse anti-total CD44 IgG recognized all isoforms of CD44. Immunolabeling with mouse anti-total CD44 IgG showed that CD44 is mainly expressed in the epithelial lining of the colon (i.e., colonocytes) of both precancerous and healthy mucosa (Figure 5; upper panel). Counterstaining with DAPI and overlaying the images shows that fibroblasts and other cells contributing to colon mucosa do not express any significant amount of CD44 that could falsely affect our results that we ascribe to colonocytes. We also immunolabelled tissue sections with anti-CD44v10 IgG to see where CD44v8-10 localizes in colon polyps (Figure 5; bottom

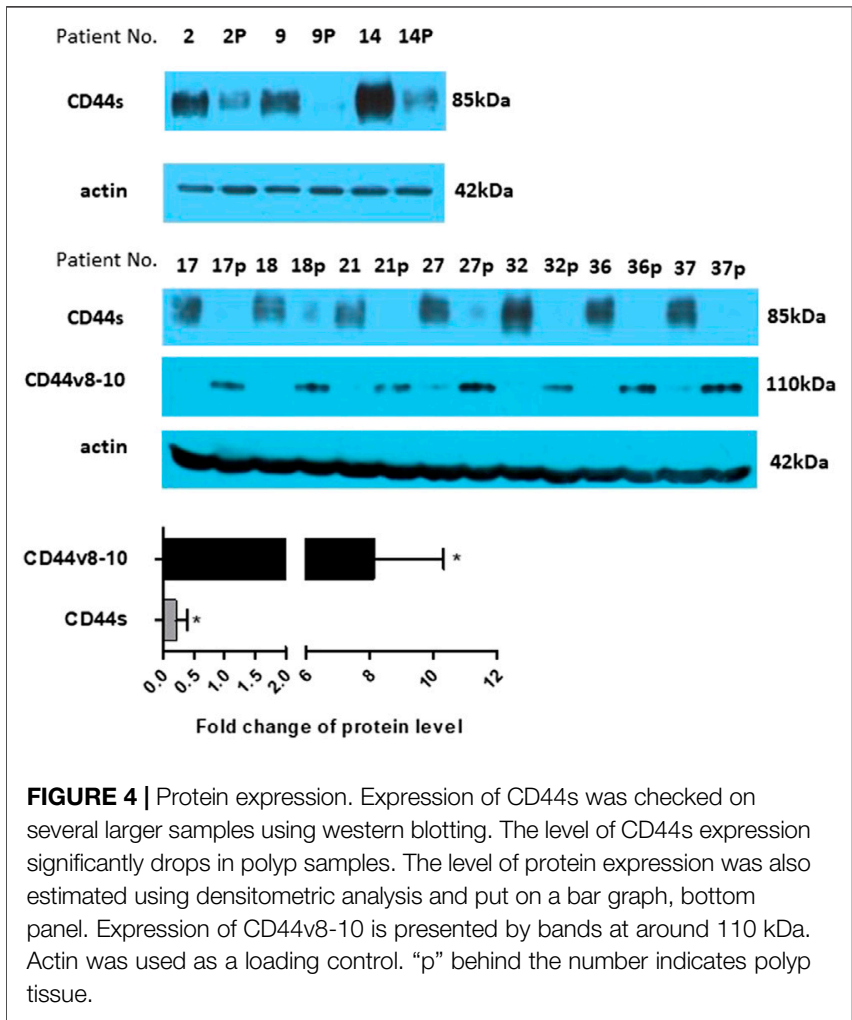

panel). The localization of CD44v8-10 was the same as demonstrated with anti-total CD44, i.e., in the epithelial lining of the colon polyps. Staining with secondary antibodies conjugated to FITC was used as a negative control of nonspecific interaction of the labeled antibodies with the tissues.

To shed more light on the mechanisms running in the background of our observation, i.e., overexpression of CD44v8-10 in colon polyps, we tested expression levels of RNA binding proteins ESRP-1 and ESRP-2 that are known to control ASP of CD44 in prostate epithelial cells [34] (Figure 6D). In conjunction with our previous work (Figure 6D), we also tested expression levels of SR-proteins SFRS6, TRA2B and Sam68 that are also known to affect alternative splicing of CD44 [6].

Only ESRP-1 gene was significantly upregulated in colon polyps (Figure 6A). We have not detected upregulation of ESRP-2 nor other SR-like proteins that have been previously documented to be involved in alternative splicing of CD44 gene (Figure 6B) [6]. Our data indicated that an ESRP-1 dependent isoform switching mechanism misprint might be running in the background of ASP in colon polyps. Not surprisingly ESRP-1 is not upregulated in colon polyps of those patients who express the lowest levels of CD44v8-10 (Figure 6C).

One of the main roles of CD44 receptor is its adhesion to extracellular matrix, mainly hyaluronic acid, HA. We tested affinity of the cells extracted from adenomatous and normal tissue samples to fluorescently labeled hyaluronic acid a natural ligand of CD44 receptor. Flow cytometry analysis showed higher fluorescence intensity of cells extracted from normal colon tissue compared to fluorescence intensity of cells extracted from colon 


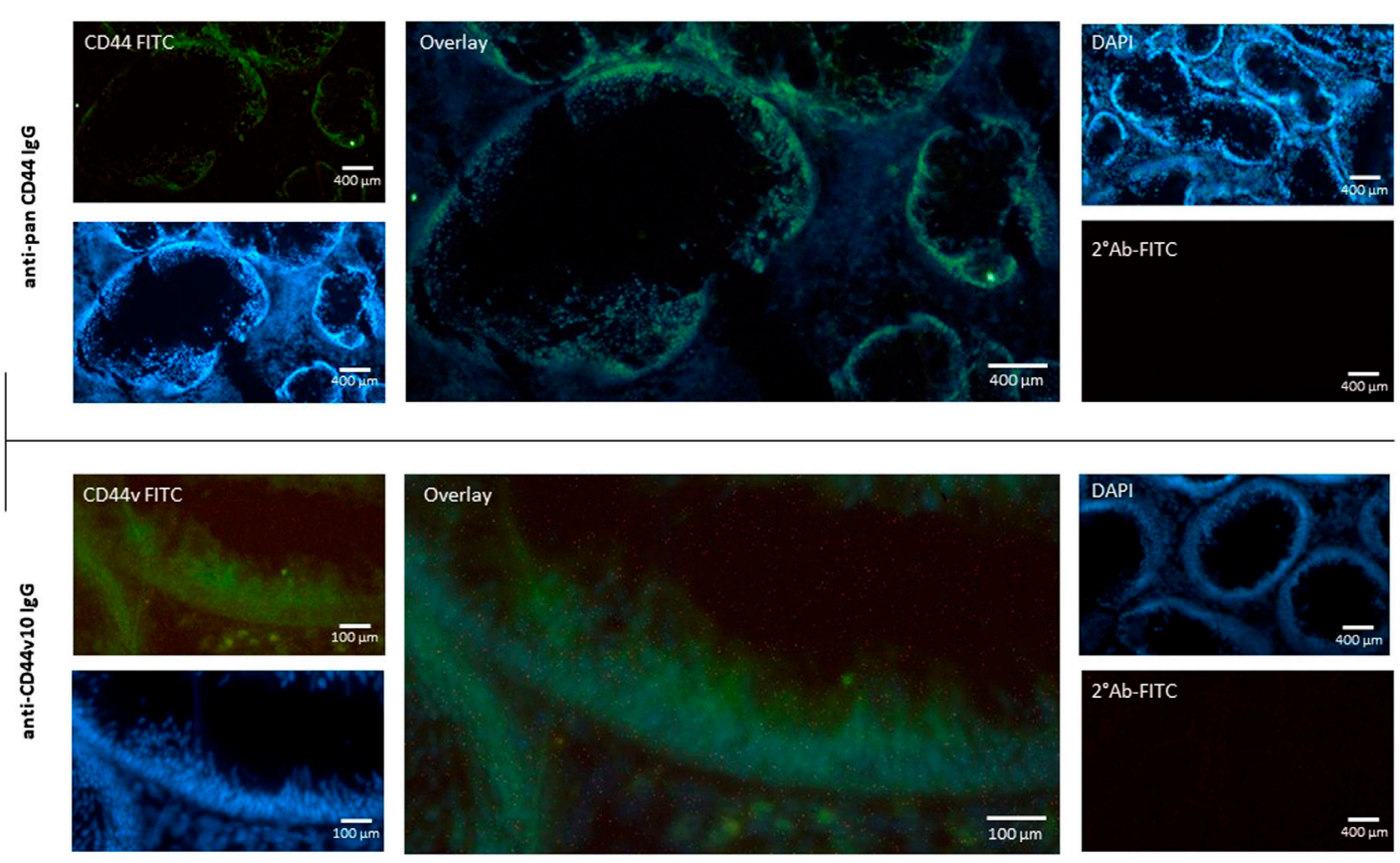

FIGURE 5 | Expression of CD44 receptor in the mucosa of adenomatous polyp. Biopsy specimens of colon polyp were frozen sectioned and immunolabeled using pan specific anti-CD44 and anti-CD44V10 lgG. Nuclei were stained with DAPI (Blue). Nonspecific binding of secondary FITC IgG was checked (upper right panel). The data indicate that the most of CD44 protein is expressed in the epithelial cells of the colon crypt base.

polyps (Figure 7). We also used CD44 non expressing T47D cell line as a negative control and $\mathrm{H} 1299$ cell line as a positive control for HA binding as in our recently published study of targeting CD44 expressing cancer cells [35]. Our results show that aberrant alternative splicing of CD44 in colon polyps might affect affinity of colonocytes to HA, the main component of extracellular matrix.

\section{DISCUSSION}

The role of CD44 and its isoforms (CD44v) as markers of malignancy or patient's prognosis remains unclear. Back in the early 90's an early work of Wielenga et al. [36] reported higher expression of v7, v8-10, v6 and v5 isoforms throughout the progression of CRC [36]. Almost twenty ears later Kopp et al. [37] made a similar observation based on qualitative data, however immunohistology and protein level assays were missing. They remained the only ones who reported expression of CD44v8-10 in early adenoma. The authors did not relate their data to adjacent normal tissue neither suggested any mechanism running in the background of the process and thus the information is incomplete from our perspective. Moreover, the molecular processes in adenomatous mucosa were overshadowed by the main message regarding isoform switching during progression of CRC. Our study is strictly focused on precancerous tissues and compares splicing events in polyp and adjacent healthy mucosa within the same patient. We have revealed significant differences in alternative splicing of
CD44 between healthy and precancerous tissue which is a novel observation. Alternative splicing of CD44 generates high numbers of variable isoforms. We used end point PCR in conjunction with agarose gel electrophoresis to positively identify expressed isoforms that became a subject of further research. We detected expression of CD44v8-10 isoform in all samples of colorectal adenoma biopsy specimen which is in accordance with a previous study [36] while only a few samples representing normal adjacent colon mucosa expressed CD44v8-10. The qRT PCR confirmed our observation, enabled quantification of the difference and allowed statistical analysis of the rate of overexpression of CD44v8-10 in colon polyps. Moreover, we proved that RNA splicing translates proportionally into the protein level, which might be a crucial condition for developing a protein based diagnostic approach. We used immunohistochemistry for identification of the site of origin of CD44 isoforms and RNA transcripts. Our data showed that all types of colorectal adenomas overexpress CD44v8-10 in the epithelial lining of the colon mucosa.

The majority of colorectal cancers arise via the conventional adenoma-carcinoma pathway while $10-20 \%$ of CRC arise from serrated lesions [38]. Serrated lesions follow a different pathway mostly due to microsatellite instability and BRAF/KRAS mutations. Conventional pathway is based on APC/KRAS/p53 mutation and chromosomal instability. Thus, serrated adenomas and conventional adenomas should be recognized as two biologically distinct subtypes of colorectal adenoma [39]. Diagnostic molecular markers should differ between these two subtypes. However, our data clearly indicate that v8-10 variant 

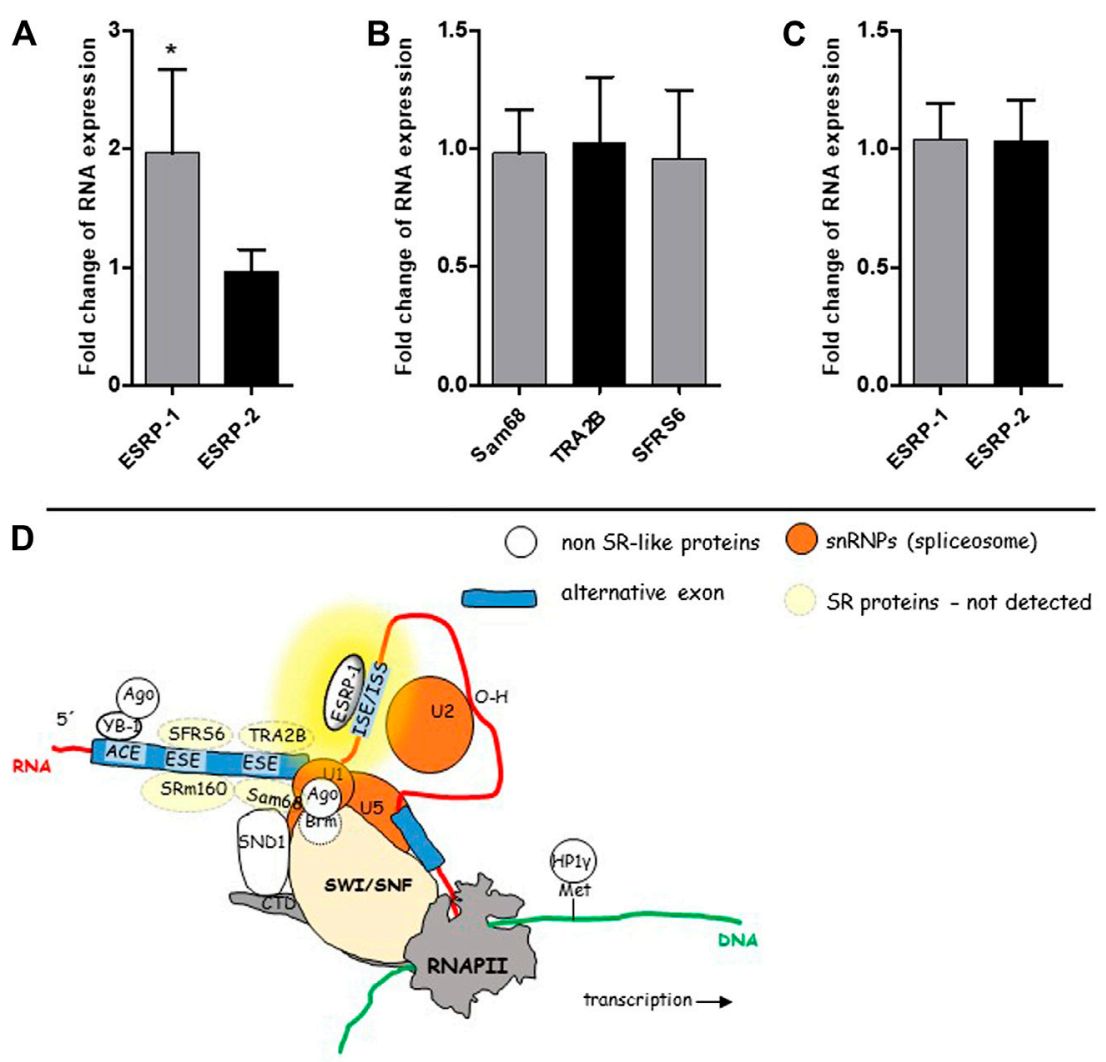

FIGURE 6 | mRNA levels of some known splicing regulators. SR-like proteins and non SR-like ESRPs were tested in the cohort of 50 patients using real time PCR. ESRP-1 was almost two-fold overexpressed in colon polyps in 50 patients (A). Expression level of ESRP-2 was similar in both types of colon mucosa. Relative expression levels of SR-like proteins in colon polyps compared to normal mucosa (B). Relative expression levels of ESRP-1 and ESRP-2 in three patients whose polyps had the lowest rate of alternative splicing (C). A graphical overview of suggested mechanism running in the background of ASP of CD44 in colon polyps (D). The yellow background color highlights the site responsible for aberrant alternative splicing. The scheme was taken from our previous publication [6].

isoform of CD44 is an exemption and is present in each subtype of colonic precancerosis and therefore could be considered as a diagnostic marker of any type of colonic precancerous lesion if proper detection technique of CD44v8-10 is developed. We found overexpression of CD44v8-10 isoform in $92 \%$ of patients with polyps and strong downregulation of CD44s in all polyp samples which is in contrast to previous study [37] where CD44s was simply detected in every adenoma sample. This huge discrepancy between the two observations might be due to the fact that we quantitatively compared relative expression of CD44s in matched tissues while Kopp et al. qualitatively detected mRNA of CD44s without any quantification. Interestingly, only $36 \%$ positive fecal occult blood tests in the same cohort of patients were detected. The percentage of positive fecal occult blood tests within the cohort of patients participating in our study is similar to observations of others [40, 41]. This further highlights potential of CD44v8-10 as a diagnostic marker. CD44v8-10 has been already identified as gastric cancer stem cell marker [21]. Indeed, future of such diagnostic markers like CD44v8-10 seems to be very much predetermined and limited by available molecular diagnostic techniques. Detection of splice variants of CD44 in body fluids is already happening in several cancers and diseases like bladder cancer, endometriosis, gastric cancer and colon cancer $[27,42,43]$ on the other hand detection of CD44 levels in healthy and oral SCC patients saliva and serum was found inconclusive [44]. Our future research will focus on possible detection of CD44v8-10 and CD44s in exfoliated cells isolated from stool.

Our data are in accord with a study of Zeilstra et al. who distinguished particular isoforms and where CD44v was shown to be important for intestinal tumorigenesis of mice while CD44s was not $[45,46]$. It is unclear whether CD44s plays any role in intestinal tumor progression [47]. Our data indicate that finding and conclusions regarding the role of CD44 and its variable isoforms in intestinal tumorigenesis can be transferred into humans. Our data further support the notion that every isoform of CD44 plays different physiological role in the tissue specific manner and should be monitored. Importance of monitoring of isoform switching is further supported by studies where CD44 isoform status serves as a response prediction marker in CD44-HA interaction based therapy where CD44 antagonist (RG7356, Roche) is tested [48, 49].

We also show that polyp-origin colonocytes in the patient No. 32 have slightly lower affinity for HA, the major component of extracellular matrix probably due to altered expression of CD44s 


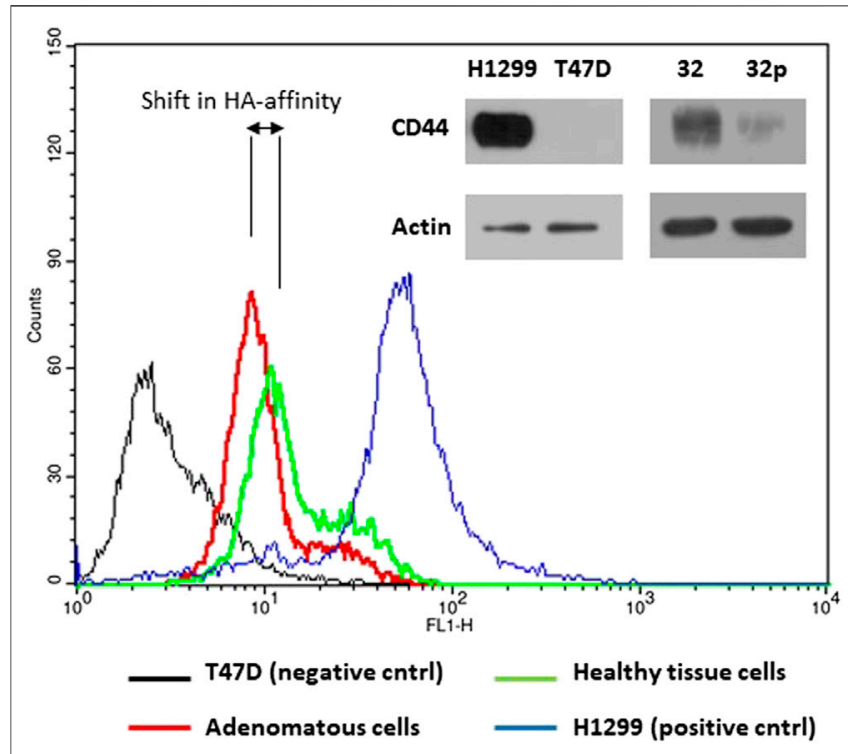

FIGURE 7 | HA-FITC binds to CD44 receptor. The upper right panel of immunoblots shows CD44s protein expression in H1299, T47D cells and cells extracted from adenomatous (32p) and healthy colon (32) mucosa using antiCD44 lgG and anti-actin lgG as a loading control. The part of the verification western blot was also published in Ref. 35 . The histograms show fluorescence intensity of cells incubated with FITC labelled hyaluronan, natural ligand of CD44s receptor. CD44 non-expressing T47D cells were used as a negative control (black line); H1299 cells were used as a positive control for CD44-HA interaction (blue line); Cells extracted from the colon polyp (red line); cells extracted from healthy colon mucosa (green line). The histogram images are representatives of three independent experiments.

(Figure 7). It is also well known that CD44v8-10 isoform does not bind HA [50,51]. We shed light on the processes running in the background of deregulation of alternative splicing of CD44 (Figure 6D). Non SR-like protein, ESRP-1, was suggested as a responsible regulator. This result is in accord with previous studies where ESRP proteins regulate variable exon inclusion into CD44 transcript [34]. We have set basis for the next set of studies focusing on mechanisms and applicability of our results in colon precancerosis screenings.

Our future research will focus on evaluation of diagnostic potential of CD44 and its isoforms mainly CD44v8-10 for early diagnosis of colon precancerosis and risk prognosis of colon cancer. Moreover, studies on isoforms of CD44 are of importance for development of hyaluronic acid targeted nanocarriers for anticancer drugs [35].

\section{REFERENCES}

1. Ferlay J, Steliarova-Foucher E, Lortet-Tieulent J, Rosso S, Coebergh JWW, Comber $\mathrm{H}$, et al. Cancer incidence and mortality patterns in Europe: estimates for 40 countries in 2012. Eur J Cancer (2013). 49(6):1374-403. doi:10.1016/j.ejca.2012.12.027

2. Torre LA, Bray F, Siegel RL, Ferlay J, Lortet-Tieulent J, Jemal A. Global cancer statistics, 2012. CA Cancer J Clin (2012). 65(2):87-108. doi:10.3322/caac.21262

3. Vogelstein B, Fearon ER, Hamilton SR, Kern SE, Preisinger AC, Leppert M, et al. Genetic alterations during colorectal-tumor development. N Engl J Med (1988). 319(9):525-32. doi:10.1056/nejm198809013190901

\section{DATA AVAILABILITY STATEMENT}

The original contributions presented in the study are included in the article, further inquiries can be directed to the corresponding authors.

\section{ETHICS STATEMENT}

The studies involving human participants were reviewed and approved by the ethical committee of the University Hospital Brno. The patients/participants provided their written informed consent to participate in this study.

\section{AUTHOR CONTRIBUTIONS}

MD: Conception and design of the study, sampling, generation, collection and analysis of data, funding; FH: Generation of data; PT-K: Generation of data; JM: Generation of data; RK: Sampling; MR: Study supervision; JT: Study supervision, funding, contribution to design of the study, approval of the manuscript; LP: Guarantor of the article; Conception and design of the study, generation, assembly and interpretation of data, data analysis, writing of the manuscript.

\section{FUNDING}

This work was supported by the following grants: the Ministry of Education, Youth and Sports OPVVV PO1 project "FIT" CZ.02.1.01/0.0/0.0/15_003/0000495 and CEREBIT CZ.02.1.01/ $0.0 / 0.0 / 16 \_025 / 0007397$ (to JT, MR); the Ministry of Agriculture of the Czech Republic MZE-RO0518 (JT). The Ministry of Health, Czech Republic, conceptual development of research organization ( $\mathrm{FnBr}, 65269705$ to MD, RK). Access to instruments in the laboratory NanoPharm (join project of Veterinary Research Institute and International Clinical Research Center (FNUSA-ICRC) (JT) is acknowledged.

\section{CONFLICT OF INTEREST}

The authors declare that the research was conducted in the absence of any commercial or financial relationships that could be construed as a potential conflict of interest.

4. Tomasetti C, Marchionni L, Nowak MA, Parmigiani G, Vogelstein B. Only three driver gene mutations are required for the development of lung and colorectal cancers. Proc Natl Acad Sci USA (2015). 112(1):118-23. doi:10.1073/pnas. 1421839112

5. Zavoral M, Suchanek S, Majek O, Fric P, Minarikova P, Minarik M, et al. Colorectal cancer screening: 20 years of development and recent progress. World J. Gastroenterol (2014). 20(14):3825-34. doi:10.3748/wjg.v20.i14.3825

6. Prochazka L, Tesarik R, Turanek J. Regulation of alternative splicing of CD44 in cancer. Cell Signal (2014). 26(10):2234-9. doi:10.1016/j.cellsig.2014.07.011

7. Toole BP. Hyaluronan-CD44 interactions in cancer: paradoxes and possibilities. Clin Cancer Res (2009). 15(24):7462-8. doi:10.1158/1078-0432.ccr-09-0479 
8. Toole BP. Hyaluronan: from extracellular glue to pericellular cue. Nat Rev Cancer (2004). 4(7):528-39. doi:10.1038/nrc1391

9. Orian-Rousseau V, Ponta H. Perspectives of CD44 targeting therapies. Arch Toxicol (2015). 89(1):3-14. doi:10.1007/s00204-014-1424-2

10. Al-Hajj M, Wicha MS, Benito-Hernandez A, Morrison SJ, Clarke MF. Prospective identification of tumorigenic breast cancer cells. Proc Natl Acad Sci (2003). 100(7):3983-8. doi:10.1073/pnas.0530291100

11. Takeo K, Kawai T, Nishida K, Masuda K, Teshima-Kondo S, Tanahashi T, et al. Oxidative stress-induced alternative splicing of transformer $2 \beta$ (SFRS10) and CD44 pre-mRNAs in gastric epithelial cells. Am J Physiology-Cell Physiol (2009). 297(2):C330-C338. doi:10.1152/ajpcell. 00009.2009

12. Batsché E, Yaniv M, Muchardt C. The human SWI/SNF subunit Brm is a regulator of alternative splicing. Nat Struct Mol Biol (2006). 13(1):22-9. doi:10. 1038/nsmb1030

13. Cappellari M, Bielli P, Paronetto MP, Ciccosanti F, Fimia GM, Saarikettu J, et al. The transcriptional co-activator SND1 is a novel regulator of alternative splicing in prostate cancer cells. Oncogene (2014). 33(29):3794-802. doi:10. 1038/onc. 2013.360

14. Ameyar-Zazoua M, Rachez C, Souidi M, Robin P, Fritsch L, Young R, et al. Argonaute proteins couple chromatin silencing to alternative splicing. Nat Struct Mol Biol (2012). 19(10):998-1004. doi:10.1038/nsmb.2373

15. Orian-Rousseau V, Chen L, Sleeman JP, Herrlich P, Ponta H. CD44 is required for two consecutive steps in HGF/c-Met signaling. Genes Dev (2002). 16(23): 3074-86. doi:10.1101/gad.242602

16. Brown RL, Reinke LM, Damerow MS, Perez D, Chodosh LA, Yang J, et al. CD44 splice isoform switching in human and mouse epithelium is essential for epithelial-mesenchymal transition and breast cancer progression. J Clin Invest (2011). 121(3):1064-74. doi:10.1172/jci44540

17. Rudy W, Hofmann M, Schwartz-Albiez R, Zöller M, Heider KH, Ponta H, et al. The two major CD44 proteins expressed on a metastatic rat tumor cell line are derived from different splice variants: each one individually suffices to confer metastatic behavior. Cancer Res (1993). 53(6):1262-8.

18. Seiter S, Arch R, Reber S, Komitowski D, Hofmann M, Ponta H, et al. Prevention of tumor metastasis formation by anti-variant CD44. J Exp Med (1993). 177(2):443-55. doi:10.1084/jem.177.2.443

19. Tanabe KK, Ellis LM, Saya H. Expression of CD44R1 adhesion molecule in colon carcinomas and metastases. The Lancet (1993). 341(8847):725-6. doi:10. 1016/0140-6736(93)90490-8

20. Günthert U, Hofmann M, Rudy W, Reber S, Zöller M, Haußmann I, et al. A new variant of glycoprotein $\mathrm{CD} 44$ confers metastatic potential to rat carcinoma cells. Cell (1991). 65(1):13-24. doi:10.1016/0092-8674(91) 90403-1

21. Lau WM, Teng E, Chong HS, Lopez KAP, Tay AYL, Salto-Tellez M, et al. CD44v8-10 is a cancer-specific marker for gastric cancer stem cells. Cancer Res (2014). 74(9):2630-41. doi:10.1158/0008-5472.can-13-2309

22. Jiang H, Zhao W, Shao W. Prognostic value of CD44 and CD44v6 expression in patients with non-small cell lung cancer: meta-analysis. Tumor Biol (2014). 35(8):7383-9. doi:10.1007/s13277-014-2150-3

23. Yan Y, Zuo X, Wei D. Concise review: emerging role of CD44 in cancer stem cells: a promising biomarker and therapeutic target. Stem Cell Transl Med (2015). 4(9):1033-43. doi:10.5966/sctm.2015-0048

24. Gao AC, Lou W, Dong JT, Isaacs JT. CD44 is a metastasis suppressor gene for prostatic cancer located on human chromosome 11p13. Cancer Res (1997). 57(5):846-9.

25. Neumayer R, Rosen HR, Reiner A, Sebesta C, Schmid A, Tüchler H, et al. CD44 expression in benign and malignant colorectal polyps. Dis Colon Rectum (1999). 42(1):50-5. doi:10.1007/bf02235182

26. Asao T, Nakamura J-i., Shitara Y, Tsutsumi S, Mochiki E, Shimura T, et al. Loss of standard type of CD44 expression in invaded area as a good indicator of lymph-node metastasis in colorectal carcinoma. Dis Colon Rectum (2000). 43(9):1250-4. ;discussion 1254-5. doi:10.1007/bf02237430

27. Miyake H, Eto H, Arakawa S, Kamidono S, Hara I. Over expression of CD44V8-10 in urinary exfoliated cells as an independent prognostic predictor in patients with urothelial cancer. J Urol (2002). 167(3):1282-7. doi:10.1016/s0022-5347(05)65282-2

28. Levine JS, Ahnen DJ. Adenomatous polyps of the colon. N Engl J Med (2006). 355(24):2551-7. doi:10.1056/nejmcp063038
29. Risio M. Reprint of: the natural history of adenomas. Best Pract Res Clin Gastroenterol (2010). 24(4):397-406. doi:10.1016/j.bpg.2010.08.002

30. Lech G, Słotwiński R, Słodkowski M, Krasnodębski IW. Colorectal cancer tumour markers and biomarkers: recent therapeutic advances. World J. Gastroenterol (2016). 22(5):1745-55. doi:10.3748/wjg.v22.i5.1745

31. Pfaffl MW. A new mathematical model for relative quantification in realtime RT-PCR. Nucleic Acids Res (2001). 29(9):45e-45. doi:10.1093/nar/ 29.9.e45

32. Procházka L, Turánek J, Tesařík R, Knotigová P, Polášková P, Andrysík Z, et al. Apoptosis and inhibition of gap-junctional intercellular communication induced by LA-12, a novel hydrophobic platinum(IV) complex. Arch Biochem Biophys (2007). 462(1):54-61. doi:10.1016/j.abb.2007.03.021

33. Iida J, Clancy R, Dorchak J, Somiari RI, Somiari S, Cutler ML, et al. DNA aptamers against exon v10 of CD44 inhibit breast cancer cell migration. PLoS One (2014). 9(2):e88712. doi:10.1371/journal.pone.0088712

34. Warzecha CC, Sato TK, Nabet B, Hogenesch JB, Carstens RP. ESRP1 and ESRP2 are epithelial cell-type-specific regulators of FGFR2 splicing. $\mathrm{Mol} \mathrm{Cel}$ (2009). 33(5):591-601. doi:10.1016/j.molcel.2009.01.025

35. Bartheldyová E, Effenberg R, Mašek J, Procházka L, Knötigová PT, Kulich P, et al. Hyaluronic acid surface modified liposomes prepared via orthogonal aminoxy coupling: synthesis of nontoxic aminoxylipids based on symmetrically $\alpha$-branched fatty acids, preparation of liposomes by microfluidic mixing, and targeting to cancer cells expressing CD44. Bioconjug Chem. (2018). 29(7):2343-56. doi:10.1021/acs.bioconjchem. $8 \mathrm{~b} 00311$

36. Wielenga VJ, Heider KH, Offerhaus GJ, Adolf GR, van den Berg FM, Ponta H, et al. Expression of CD44 variant proteins in human colorectal cancer is related to tumor progression. Cancer Res (1993). 53(20):4754-6.

37. Kopp R, Fichter M, Schalhorn G, Danescu J, Classen S. Frequent expression of the high molecular, 673-bp CD44v3,v8-10 variant in colorectal adenomas and carcinomas. Int J Mol Med (2009). 24(5):677-83. doi:10.3892/ijmm_00000279

38. Mäkinen MJ. Colorectal serrated adenocarcinoma. Histopathology (2007). 50(1):131-50. doi:10.1111/j.1365-2559.2006.02548.x

39. Konishi K, Yamochi T, Makino R, Kaneko K, Yamamoto T, Nozawa H, et al. Molecular differences between sporadic serrated and conventional colorectal adenomas. Clin Cancer Res (2004). 10(9):3082-90. doi:10.1158/1078-0432.ccr03-0334

40. Muinuddin A, Aslahi R, Hopman WM, Paterson WG. Relationship between the number of positive fecal occult blood tests and the diagnostic yield of colonoscopy. Can J Gastroenterol (2013). 27(2):90-4. doi:10.1155/2013/ 612314

41. Wei SC, Tsao PN, Wang YT, Lin BR, Wu DC, Tsai WS, et al. Using serum placenta growth factor could improve the sensitivity of colorectal cancer screening in fecal occult blood negative population: a multicenter with independent cohort validation study. Cancer Med (2019). 8(7):3583-91. doi:10.1002/cam4.2216

42. Mashayekhi F, Aryaee H, Mirzajani E, Yasin AA, Fathi A. Soluble CD44 concentration in the serum and peritoneal fluid samples of patients with different stages of endometriosis. Arch Gynecol Obstet (2015). 292(3):641-5. doi:10.1007/s00404-015-3654-9

43. Palagani V, El Khatib M, Krech T, Manns MP, Malek NP, Plentz RR. Decrease of CD44-positive cells correlates with tumor response to chemotherapy in patients with gastrointestinal cancer. Anticancer Res (2012). 32(5):1747-55.

44. Seyedmajidi S, Seyedmajidi M, Foroughi R, Zahedpasha A, Zolfaghari Saravi Z, Pourbagher R, et al. Comparison of salivary and serum soluble CD44 levels between patients with oral SCC and healthy controls. Asian Pac J Cancer Prev (2018). 19(11):3059-63. doi:10.31557/apjcp.2018.19.11.3059

45. Zeilstra J, Joosten SPJ, Dokter M, Verwiel E, Spaargaren M, Pals ST. Deletion of the WNT target and cancer stem cell marker CD44 in Apc(Min/+) mice attenuates intestinal tumorigenesis. Cancer Res (2008). 68(10):3655-61. doi:10. 1158/0008-5472.can-07-2940

46. Zeilstra J, Joosten SPJ, van Andel H, Tolg C, Berns A, Snoek M, et al. Stem cell $\mathrm{CD} 44 \mathrm{v}$ isoforms promote intestinal cancer formation in $\mathrm{Apc}(\mathrm{min})$ mice downstream of Wnt signaling. Oncogene (2014). 33(5):665-70. doi:10.1038/ onc.2012.611

47. Guo W, Frenette PS. Alternative CD44 splicing in intestinal stem cells and tumorigenesis. Oncogene (2014). 33(5):537-8. doi:10.1038/onc.2013.260 
48. Zhang S, Wu CCN, Fecteau J-F, Cui B, Chen L, Zhang L, et al. Targeting chronic lymphocytic leukemia cells with a humanized monoclonal antibody specific for CD44. Proc Natl Acad Sci (2013). 110(15):6127-32. doi:10.1073/ pnas. 1221841110

49. Birzele F, Voss E, Nopora A, Honold K, Heil F, Lohmann S, et al. CD44 isoform status predicts response to treatment with anti-CD44 antibody in cancer patients. Clin Cancer Res (2015). 21(12):2753-62. doi:10.1158/1078-0432.ccr14-2141

50. Sy MS, Guo YJ, Stamenkovic I. Distinct effects of two CD44 isoforms on tumor growth in vivo. J Exp Med (1991). 174(4):859-66. doi:10.1084/jem. 174.4.859
51. Bartolazzi A, Peach R, Aruffo A, Stamenkovic I. Interaction between CD44 and hyaluronate is directly implicated in the regulation of tumor development. J Exp Med (1994). 180(1):53-66. doi:10.1084/jem.180.1.53

Copyright (๐ 2021 Dastych, Hubatka, Turanek-Knotigova, Masek, Kroupa, Raška, Turanek and Prochazka. This is an open-access article distributed under the terms of the Creative Commons Attribution License (CC BY). The use, distribution or reproduction in other forums is permitted, provided the original author(s) and the copyright owner(s) are credited and that the original publication in this journal is cited, in accordance with accepted academic practice. No use, distribution or reproduction is permitted which does not comply with these terms. 